\title{
Self-Powered and Low-Power Piezoelectric Vibration Control Using Nonlinear Approaches
}

\author{
Mickaël Lallart and Daniel Guyomar \\ Université de Lyon, INSA-Lyon, LGEF EA 682, F-69621, Villeurbanne
}

France

\section{Introduction}

The constant proliferation of embedded systems as well as miniaturized devices has raised the issue of efficiently limiting the vibrations and/or providing precise positioning using a very few amount of energy. A typical application example is the damping of vibrating electronic cards, in order to limit the risk of microcracks in conductive tracks. Because of the integration constraints of these systems, the use of viscoelastic materials (i.e., direct dissipation of the mechanical energy into heat (Johnson, 1995)) is not applicable. Hence, the use of energy conversion media is mandatory in this case. In particular, piezoelectric materials are good candidates for the control of embedded devices, thanks to their high power densities and integration potentials (Veley \& Rao, 1996). It should be also noted that using piezoelectric elements allows achieving various functions, such as actuation, sensing and energy harvesting (Inman, Ahmadian \& Claus, 2001; Qiu \& Haraguchi, 2006; Guyomar et al., 2007a; Lallart et al., 2008a), making them very attractive in integrated systems.

However, the use of standard active control schemes is also prohibited when dealing with integrated systems, as their high power requirements necessitate bulky amplifiers (Gerhold, 1989). Such control schemes need a full feedback loop as well, including sensors (although some control schemes can use the transducer itself as a sensor (Qiu \& Haraguchi, 2006)) and microcontrollers that may not be easily integrated.

In addition, the use of passive schemes featuring electroactive materials (i.e., shunted systems (Lesieutre, 1998)) is also quite complex, as the required components are usually difficult to implement, especially in the case of using piezoelectric actuators where the required inductance for an efficient control is usually very large (several Henrys), necessitating the use of synthetic components that have to be externally powered (Fleming and Moheimani, 2003). These control techniques have limited performance as well as high sensitivity to frequency drifts caused by temperature variations or ageing, and their implementation for the control of several modes is quite complex $(\mathrm{Wu}, 1998)$.

Hence, the combination of passive and active control schemes has been proposed in order to combine their advantages while limiting their drawbacks. In particular, it has been shown that applying nonlinear methods can lead to significant damping abilities (Clark, 2000; Davis \& Lesieutre, 2000; Cunefare, 2002; Holdhusen \& Cunefare, 2003; Wickramasinghe et al., 2004; Nitzsche et al., 2005; Makihara, Onoda \& Minesugi, 2007). However, the nature of these approaches is often complex, limiting their realistic application, especially in integrated systems.

Source: Vibration Control, Book edited by: Dr. Mickaël Lallart,

ISBN 978-953-307-117-6, pp. 380, September 2010, Sciyo, Croatia, downloaded from SCIYO.COM 
Therefore, the development of effective techniques for vibration control that can be easily integrated is an issue. The purpose of this chapter is to expose several techniques addressing this problem. In order to meet the low-power and integration constraints, the proposed approaches are based on a simple nonlinear processing of the output voltage of piezoelectric elements, resulting in a magnification of the conversion abilities of the system. Hence, this increase of the coupling coefficient allows reducing the mechanical energy, leading to a damping effect in terms of mechanical vibrations.

The chapter is organized as follows. Section 2 introduces a rough but accurate model of the behavior of an electromechanical structure near one of its resonance frequencies, as well as the basic concepts of the nonlinear treatment. The direct application of this technique for vibration damping purposes is exposed in Section 3, leading to the concept of Synchronized Switch Damping (SSD), whose principles lie in a fast piezovoltage inversion when this latter reaches either a maximum or a minimum value. The extension of this method to a nonsynchronized technique called Blind Switch Damping is then developed in Section 4. In Section 5 is proposed a similar concept than the SSD, but with a voltage switching on zero displacement values, allowing a control of the resonance frequency of the device. Finally, Section 6 concludes the chapter, recalling the main results and exposing a comparison of the advantages and drawbacks of the exposed techniques.

\section{Modeling and nonlinear conversion enhancement}

The aim of this section is to expose a simple model (developed by Badel et al. (2007)) for the description of the behavior of an electromechanical structure near one of its resonance frequencies (which correspond to the highest displacement magnitude and therefore to the cases that may be harmful to the system). This model will be used in the following sections for the theoretical developments in order to evaluate the damping abilities of the various methods.

The basic principles of the nonlinear treatment are also provided, showing the increase in terms of conversion abilities of the piezodevice.

\subsection{Electromechanical structure modeling}

For the sake of simplicity, it will be considered that the electromechanical structure is a simple cantilever beam, as depicted in Figure 1. However, it can be noted that the proposed model can also be applied to any structures, such as plates. In addition, it is assumed that:

i. $\quad$ The strain has only two dimensions (i.e., null stress along the $x_{2}$ axis).

ii. The plane sections of the beam remain plane (Euler-Bernoulli assumption), so that no shear strain appears and the strain along the $x_{3}$ axis is null.

iii. The dynamic deformed shape of the flexural mode is close to the static one.

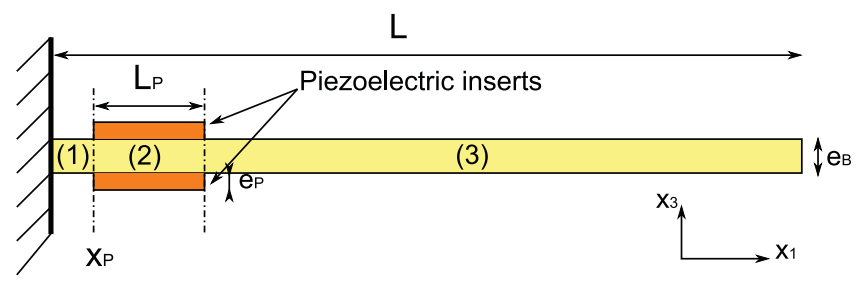

Fig. 1. Electromechanical structure 
Under these conditions, the relationship between strain $S_{1}$ and stress $T_{1}$ along the $x_{1}$ axis for the beam turns to:

$$
T_{1}=c_{B} S_{1}
$$

with $c_{B}$ the elastic rigidity of the structure in-plane strain, and equals $c_{B}=Y /(1-v)$, with $Y$ and $v$ the Young modulus and Poisson's ratio.

For the piezoelectric element, the constitutive equations are given by:

$$
\left\{\begin{array}{l}
S_{1}=s_{11}^{E} T_{1}+s_{12}^{E} T_{2}+s_{13}^{E} T_{3}+d_{13} E_{3} \\
S_{2}=s_{12}^{E} T_{1}+s_{11}^{E} T_{2}+s_{13}^{E} T_{3}+d_{13} E_{3} \\
D_{3}=\epsilon_{33}^{T} E_{3}+d_{13} T_{1}+d_{13} T_{2}+d_{33} T_{3}
\end{array}\right.
$$

where $D$ and $E$ denote the electric displacement and electric field. $s_{i j}^{E}, \epsilon_{i i}^{T}$ and $d_{i j}$ are given as the elastic constant under constant electric field, permittivity under constant stress and piezoelectric charge coefficient respectively.

According to the previous assumptions $\left(S_{2}=0\right.$ and $\left.T_{3}=0\right)$, the stress-strain relationships may be simplified as:

$$
S_{1}=\left(s_{11}^{E}-\frac{s_{12}^{E 2}}{s_{11}^{E}}\right) T_{1}+d_{13}\left(1-\frac{s_{12}^{E}}{s_{11}^{E}}\right) E_{3}
$$

When the piezoelectric element is short-circuited $\left(E_{z}=0\right)$, it is therefore possible to define a rigidity in short-circuit conditions $c_{P E}$ given by:

$$
c_{P E}=\frac{s_{11}^{E}}{s_{11}^{E 2}-s_{12}^{E 2}} .
$$

Similarly, using the expression of the electric displacement gives the rigidity in open-circuit conditions $\left(D_{3}=0\right) c_{P D}$ defined as:

$$
c_{P D}=\left[s_{11}^{E}-\frac{s_{12}^{E 2}}{s_{11}^{E}}-\frac{d_{13}^{2}}{\epsilon_{33}^{T}-\frac{d_{13}{ }^{2}}{s_{11}^{E}}}\left(1-\frac{s_{12}^{E}}{s_{11}^{E}}\right)^{2}\right]^{-1} .
$$

In addition, it has been assumed that the dynamic and static flexural mode shapes are similar. Therefore, the expression of the second order derivative of the flexural displacement yields:

$$
\frac{d^{2} u_{3}}{d x_{1}^{2}}=\frac{2 S_{1}}{e_{B}}=c_{x} I_{x}
$$

where: 


$$
c_{x} I_{x}=\left\{\begin{array}{ll}
c_{B} I_{B} & \text { in non-piezoelectric areas (zones (1) and (3)) } \\
c_{B} I_{B}+c_{P D} I_{P} & \text { in piezoelectric areas (zone (2)) }
\end{array},\right.
$$

with $I_{B}$ and $I_{P}$ respectively referring to the beam and piezoelectric moments of inertia. Hence, by integrating Eq. (7) and performing an energy analysis ${ }^{1}$, it is possible to define four stiffnesses:

$$
\left\{\begin{array}{ll}
K_{B 1}=\frac{3 C_{B} I_{B}}{L^{3}-\left(L-x_{P}\right)^{3}} & \text { for the beam in zone (1) } \\
K_{B 2}=\frac{3 C_{B} I_{B}}{\left(L-x_{P}\right)^{3}-\left(L-L_{P}-x_{P}\right)^{3}} & \text { for the beam in zone (2) } \\
K_{B 3}=\frac{3 C_{B} I_{B}}{\left(L-L_{P}-x_{P}\right)^{3}} & \text { for the beam in zone (3) } \\
K_{P E}=\frac{3 C_{P E} I_{P}}{\left(L-L_{P}-x_{P}\right)^{3}} & \text { for the piezoelectric element } \\
\text { (short-circuited) in zone (2) }
\end{array},\right.
$$

as well as a dynamic mass $M$ of the structure:

$$
M=\frac{w}{\left.u_{3}\right|_{L} ^{2}}\left[\int_{0}^{L}\left(e_{B} \rho_{B}\right) d x+2 \int_{x_{P}}^{x_{P}+L_{P}}\left(e_{P} \rho_{P}\right) d x\right]
$$

with $w$ the width of the beam (and piezoelectric insert) and $\rho_{B}$ and $\rho_{P}$ the density of the beam and of the piezoelectric element.

The piezoelectric effect in terms of electromechanical coupling may also be obtained by integrating in the space domain the time derivative of the constitutive equation giving the electrical displacement $D_{3}$ as a function of the electric field $E_{3}$ and strain $S_{1}$, whose expression under the considered assumptions is given by:

$$
D_{3}=\epsilon_{33}^{S} E_{3}+e_{33} S_{3},
$$

yielding the expression of the current $I$ flowing out of the insert:

$$
I=\alpha_{P}\left(\dot{u}_{2}-\dot{u}_{1}\right)-C_{0 P} \dot{V} \text { with } \alpha_{P}=-\frac{2 e_{33} w L_{P}}{e_{P}}>0 \text { and } C_{0 P}=\frac{2 \epsilon_{33}^{S} w L_{P}}{e_{P}},
$$

with $\dot{u}_{1}$ and $\dot{u}_{2}$ denoting the velocities at the length $x_{P}$ and $x_{P}+L_{P}$, and $V$ the piezovoltage. In addition, the effect of the piezoelectric element in terms of mechanical behavior may be obtained from the expression of the stress along the $x_{3}$ direction:

$$
T_{3}=c_{33}^{E} S_{3}-e_{33} E_{3},
$$

leading to the expression of the force $F_{P}$ exerted by the piezoelectric element:

\footnotetext{
${ }^{1}$ For the entire derivation, the reader may refer to Badel et al. (2007).
} 


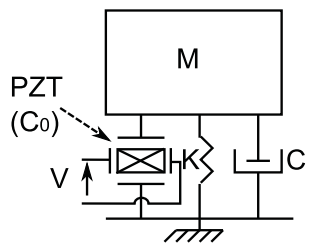

Fig. 2. SDOF model of the electromechanical structure

$$
F_{P}=K_{P E}\left(u_{2}-u_{1}\right)+\alpha_{P} V .
$$

It is however possible to simplify the model as a Single Degree of Freedom (SDOF) electromechanically coupled spring-mass-damper system as illustrated in Figure 2, yielding the global electromechanical equation set:

$$
\left\{\begin{array}{c}
M \ddot{u}+C \dot{u}+K_{E} u=F-\alpha V \\
I=\alpha \dot{u}-C_{0} \dot{V}
\end{array},\right.
$$

with $u$ the displacement and $F$ the applied force at a given point and where $K_{E}, \alpha$ and $C_{0}$ are the global short-circuit stiffness, force factor and clamped capacitance:

$$
\left\{\begin{array}{l}
K_{E}=\frac{K_{B 1}\left(K_{B 2}+K_{P E}\right) K_{B 3}}{K_{B 1}\left(K_{B 2}+K_{P E}\right)+\left(K_{B 2}+K_{P E}\right) K_{B 3}+K_{B 1} K_{B 3}} \\
\alpha=\frac{\alpha_{P} K_{B 1} K_{B 3}}{K_{B 1}\left(K_{B 2}+K_{P E}\right)+\left(K_{B 2}+K_{P E}\right) K_{B 3}+K_{B 1} K_{B 3}} \\
C_{0}=C_{0 P}+\frac{\alpha_{P}{ }^{2}\left(K_{B 1}+K_{B 3}\right)}{K_{B 1}\left(K_{B 2}+K_{P E}\right)+\left(K_{B 2}+K_{P E}\right) K_{B 3}+K_{B 1} K_{B 3}}
\end{array} .\right.
$$

$C$ is defined as the structural damping coefficient, whose value is obtained from the mechanical quality factor $Q_{M}$ of the structure at the considered mode in open-circuit condition:

$$
C=\frac{\sqrt{K_{D} M}}{Q_{M}},
$$

with $K_{D}$ the open-circuit equivalent stiffness:

$$
K_{D}=K_{E}+\frac{\alpha^{2}}{C_{0}} .
$$

The global electromechanical coupling coefficient $k$, which gives the ability of the piezomaterial for converting the mechanical energy into electric energy (and conversely), can also be obtained from the model parameters as:

$$
k^{2}=1-\frac{f_{E}^{2}}{f_{D}{ }^{2}}=\frac{\alpha^{2}}{\alpha^{2}+C_{0} K_{E}}=\frac{\alpha^{2}}{C_{0} K_{D}}
$$

with $f_{E}$ and $f_{D}$ the short-circuit and open-circuit resonance frequencies. 


\subsection{Nonlinear conversion enhancement}

From the mechanical equation of the model (Eq. (14)), it is possible to write the energy balance over a time period $\left[t_{1} ; t_{1}+\tau\right]$ as:

$$
\frac{1}{2} M\left[\dot{u}^{2}\right]_{t_{1}}^{t_{1}+\tau}+C \int_{t_{1}}^{t_{1}+\tau} \dot{u}^{2} d t+\frac{1}{2} K_{E}\left[u^{2}\right]_{t_{1}}^{t_{1}+\tau}=\int_{t_{1}}^{t_{1}+\tau} F \dot{u} d t-\alpha \int_{t_{1}}^{t_{1}+\tau} V \dot{u} d t
$$

where the left side members are respectively the kinetic energy, dissipated energy and potential energy. The left side terms correspond to the provided energy and converted energy. According to the electrical equation of Eq. (14), this latter can be decomposed into:

$$
\alpha \int_{t_{1}}^{t_{1}+\tau} V \dot{u} d t=\frac{1}{2} C_{0}\left[V^{2}\right]_{t_{1}}^{t_{1}+\tau}+\int_{t_{1}}^{t_{1}+\tau} V I d t
$$

where the first term of the right side members is the electrostatic energy available on the piezoelectric element, and the second term the energy exchanged with the control system.

Hence, from Eq. (19), it can be shown that in order to increase the part of converted energy, it is possible to:

1. Increase the voltage

2. Reduce the phase shift between the voltage and velocity

Both of these solutions can be achieved using a simple nonlinear treatment that does not require any external energy. The principles of this approach consist in quickly inverting the voltage when this latter reaches a maximum or a minimum value (corresponding to a maximal electrostatic energy). This inversion is however performed in an imperfect fashion i.e., the ratio of the absolute voltage values after and before the inversion is equal to $\gamma$, with $0 \leq \gamma \leq 1$.

Thanks to the continuity of the voltage ensured by the dielectric behavior of the piezoelectric element, this leads to a cumulative effect that allows a significant increase of the voltage (Figure 3). In addition, such a process also splits the voltage into two components: one that is proportional to the displacement (as in open-circuit conditions with zero initial voltage and displacement), and one created by the inversion process that is a piecewise constant function proportional to the speed ${ }^{2}$. As this latter is usually much larger than the voltage produced by the open-circuit condition, the voltage is almost proportional to the speed sign. Hence, both of the conversion enhancement possibilities are met using a simple nonlinear treatment.

The way the voltage inversion can be achieved is very simple as well. It consists in using a resonant electrical network for performing the inversion process. This resonant network is composed of an inductance $L$ and the piezoelectric capacitance $\left(C_{0}\right)$. Each time the voltage reaches a maximum or minimum value, the inductance is connected to the piezoelement (that has an initial charge $q_{0}$ ) through a digital switch $S W$ (Figure 4). Hence, the voltage starts oscillating around 0 . In particular, after half a period of this electrical oscillation, the voltage is inverted. At this time the digital switch is opened, disconnecting the inductance from the piezoelement which is therefore in open circuit condition. The total switching time $t_{s w}$ is thus given by:

\footnotetext{
2 or, in monochromatic excitation, in phase with the speed
} 


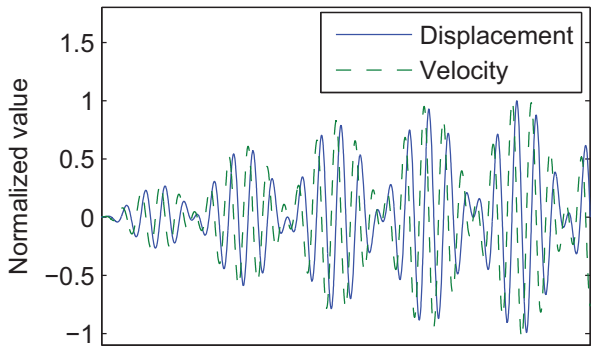

Time (arbitrary unit)

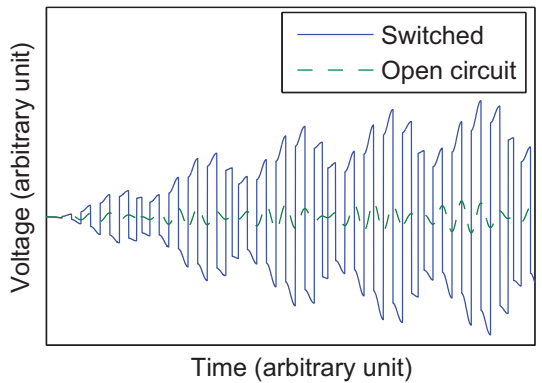

Fig. 3. Conversion enhancement principles using nonlinear approach based on voltage inversion
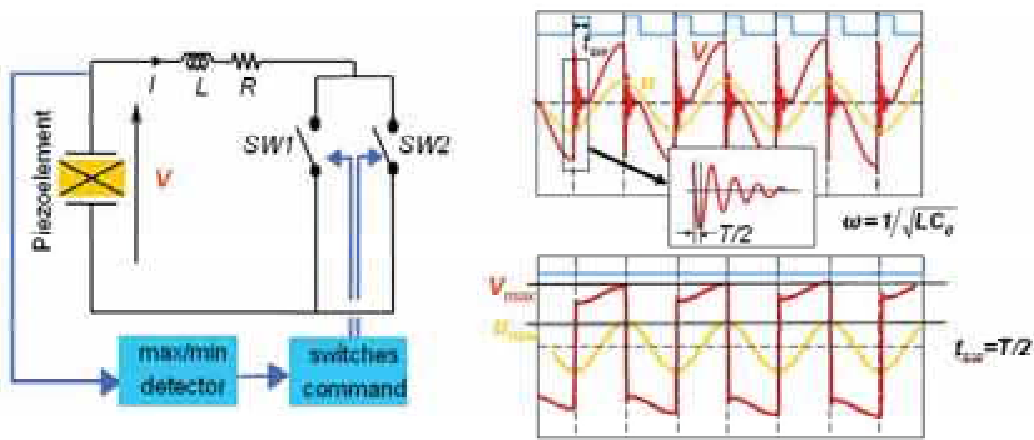

Fig. 4. Practical implementation of the voltage inversion

$$
t_{s w}=\pi \sqrt{L C_{0}} .
$$

However, because of losses in the switching circuit (modeled by a resistance $R$ ), the inversion is not perfect and characterized by the inversion coefficient $\gamma$ defined as:

$$
\gamma=e^{-R \frac{\pi}{2} \sqrt{\frac{C_{0}}{L}}}
$$

From an analysis of the maximal available electrostatic energy (given as the half product of the piezocapacitance with the squared maximal voltage), it can be shown that the electrical energy (under constant displacement magnitude) is increased by a typical factor from 12 to 200 when using such a nonlinear approach.

Many applications may benefit from this conversion ability increase, the most significative being energy harvesting from mechanical solicitations (Guyomar et al., 2005; Lefeuvre et al., 2006a; Lallart \& Guyomar, 2008b; Lallart et al., 2008c) and vibration control (e.g., for anechoic purposes (Guyomar et al., 2006a)).

\section{Synchronized Switch Damping (SSD) techniques}

Now the basic modeling and nonlinear conversion enhancement exposed, it is proposed in this section to directly apply the proposed concept to damping purposes. 
In addition, it will be considered that voltage sources may be added in the circuit in order to compensate the losses during the inversion process (Figure 5) ${ }^{3}$. Nevertheless, the addition of this external power supply makes the technique no longer semi-passive, as external energy is provided to active element (this energy remains nevertheless small). Hence, according to the value of the components, the different obtained techniques are summarized in Table 1 and depicted in Figure 6.

\subsection{Performance under monochromatic excitation}

In a first approach, it is proposed to evaluate the performance of the SSD techniques under a monochromatic excitation at one of the resonance frequencies of the system. For the sake of conciseness, the following theoretical development will be made considering all the parameters appearing in Figure 5, the damping abilities of a particular technique being obtained by replacing the parameters by those listed in Table 1.

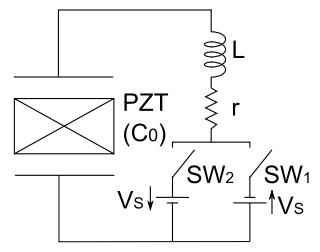

Fig. 5. General schematic for the SSD damping techniques
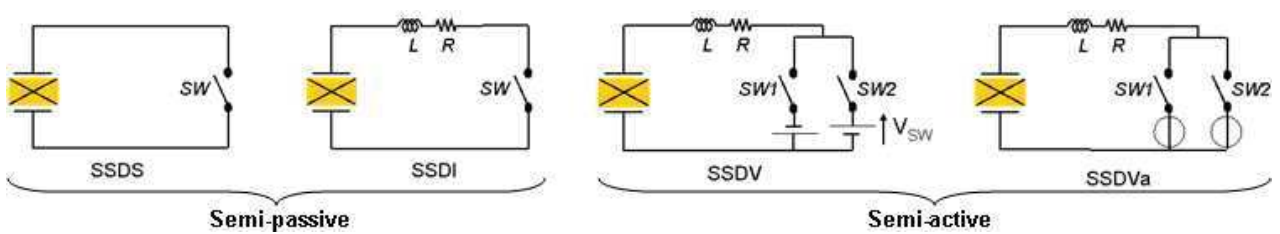

Fig. 6. SSD technique schematics

\begin{tabular}{ll|ccc} 
Designation & Kind & $\begin{array}{c}\text { Inversion } \\
\text { factor }\end{array}$ & $\begin{array}{c}\text { Voltage } \\
\text { source }\end{array}$ \\
\hline SSDS (Richard et al., & $\begin{array}{l}\text { (Synchronized Switch } \\
\text { Damping on Short- circuit) } \\
\text { 1999) }\end{array}$ & Semi-passive & 0 & 0 \\
SSDI (Richard et al., & $\begin{array}{l}\text { Synchronized Switch } \\
\text { Damping on Inductor) } \\
\text { 2000; Petit et al., 2004) }\end{array}$ & $\begin{array}{l}\text { Semi-passive } \\
\text { SSDV (Lefeuvre et al., }\end{array}$ & $\gamma$ & 0 \\
2006b) & $\begin{array}{l}\text { Samphronized Switch } \text { on Voltage sources) } \\
\text { (Synchronized Switch } \\
\text { SSDVa }\end{array}$ & Semi-active & $\gamma$ & $\pm \beta \frac{\alpha}{C_{0}} u_{M}$ \\
2006) & $\begin{array}{l}\text { Damping on adaptive } \\
\text { Voltage sources) }\end{array}$ & & & \\
\hline
\end{tabular}

Table 1. SSD technique properties

\footnotetext{
${ }^{3}$ In this case, the inversion is done with respect to the value of the voltage source.

${ }^{4} \beta$ refers to the tuning coefficient and $u_{M}$ to the displacement magnitude - it can be besides shown that the voltage source tuning is equivalent to an increase of the inversion factor $\gamma$.
} 


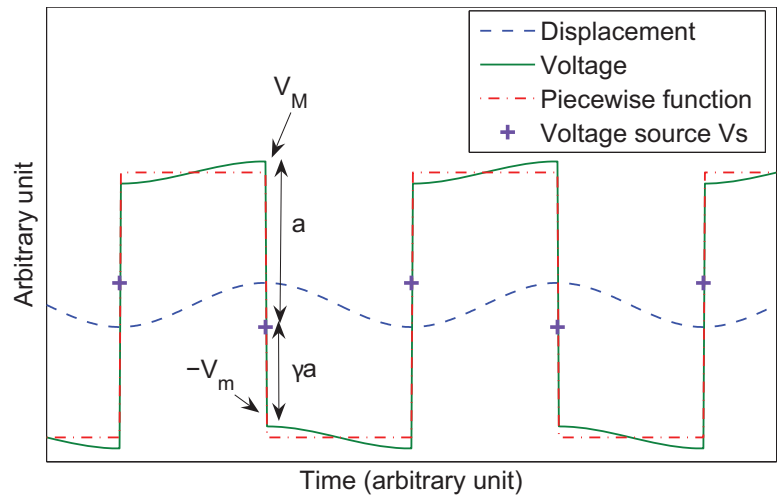

Fig. 7. Waveforms of the SSD techniques in monochromatic excitation

The theoretical performance evaluation are based on the previously exposed electromechanical model (Eq. (14)). In the case of the SSD techniques and assuming a sine excitation, the voltage maybe decomposed into two voltages - one proportional to the displacement (open-circuit voltage) and one that is proportional to the sign of the speed:

$$
V=\frac{\alpha}{C_{0}} u+H \operatorname{sign}(\dot{u}),
$$

with $H$ the magnitude of the voltage in phase with the speed, which can be found using the following relations (Figure 7):

$$
\left\{\begin{array}{c}
H=\frac{1}{2}\left(V_{M}+V_{m}\right) \\
V_{m}-V_{S}=\gamma\left(V_{M}+V_{S}\right) \\
V_{M}-V_{m}=2 \frac{\alpha}{C_{0}} u_{M}
\end{array} .\right.
$$

Hence, the amplitude of the piecewise function yields:

$$
H=\frac{1+\gamma}{1-\gamma}\left(\frac{\alpha}{C_{0}} u_{M}+V_{S}\right)
$$

Assuming that the structure filters higher harmonics, the expression of the piezovoltage may be approximated by its first harmonic, which leads to:

$$
V(\omega) \approx \frac{\alpha}{C_{0}} U(\omega)+j \frac{4}{\pi} \frac{1+\gamma}{1-\gamma}\left(\frac{\alpha}{C_{0}} U(\omega)+V_{S}\right)
$$

in the frequency domain, with $U(\omega)$ the Fourier transform of the time-domain displacement $u$ and $\omega$ the angular frequency. From this equation, it can be seen that the effect of the SSD control is equivalent to a dry friction, as the energy cycle only depends on the displacement magnitude, and not on the frequency. 
Hence, merging this expression with the motion equation of the electromechanical structure Eq. (14) yields the expression of the displacement in the frequency domain:

$$
U(\omega)=\frac{F(\omega)-j \alpha \frac{4}{\pi} \frac{1+\gamma}{1-\gamma} V_{S}}{-M \omega^{2}+j\left(C \omega+\frac{4}{\pi} \frac{1+\gamma}{1-\gamma} \frac{\alpha^{2}}{C_{0}}\right)+K_{D}} .
$$

Assuming that the structure is weakly damped, the expression of the displacement magnitude at the resonance frequency is given by:

$$
\left.\left(u_{M}\right)_{r e s}\right|_{S S D}=\frac{F_{M}-\alpha \frac{4}{\pi} \frac{1+\gamma}{1-\gamma} V_{S}}{C \omega+\frac{4}{\pi} \frac{1+\gamma}{1-\gamma} \frac{\alpha^{2}}{C_{0}}}
$$

where $F_{m}$ denotes the force magnitude. Hence, the obtained attenuation at the resonance frequency when using the SSD techniques yields:

$$
A_{S S D}=\frac{1-\alpha \frac{4}{\pi} \frac{1+\gamma}{1-\gamma} \frac{V_{S}}{F_{M}}}{\left(1+\frac{4}{\pi} \frac{1+\gamma}{1-\gamma} \frac{\alpha^{2}}{C_{0} C \omega}\right)}=\frac{1-\alpha \frac{4}{\pi} \frac{1+\gamma}{1-\gamma} \frac{V_{S}}{F_{M}}}{\left(1+\frac{4}{\pi} \frac{1+\gamma}{1-\gamma} k^{2} Q_{M}\right)}
$$

The associated attenuation of the different SSD techniques (SSDS, SSDI, SSDV, SSDVa) are listed in Table 2, and typical frequency response functions as well as associated energy cycles are depicted in Figure 8. In the case of the SSDVa technique, the value of the voltage source is matched to the vibration magnitude such as:

$$
\left|V_{S}\right|=\beta \frac{\alpha}{C_{0}} u_{M}
$$

Figure 8 demonstrates the effectiveness of the semi-passive approaches (SSDS and SSDI) for efficiently controlling the vibrations compared to the shunted approach. Especially, the addition of an inductance (SSDI approach) for performing a voltage inversion rather than a charge cancellation (SSDS technique) allows a significant enhancement of the damping abilities. In addition, the performance can be greatly improved using a few amount of external energy, as demonstrated by the transfer functions obtained in the case of

$$
\begin{array}{c|cccc}
\text { Technique } & \text { SSDS } & \text { SSDI } & \text { SSDV } & \text { SSDVa } \\
\text { Attenuation } & \frac{1}{1+\frac{4}{\pi} k^{2} Q_{M}} & \frac{1}{1+\frac{4}{\pi} \frac{1+\gamma}{1-\gamma} k^{2} Q_{M}} & \frac{1-\alpha \frac{4}{\pi} \frac{1+\gamma}{1-\gamma} \frac{V_{S}}{F_{M}}}{1+\frac{4}{\pi} \frac{1+\gamma}{1-\gamma} k^{2} Q_{M}} & \frac{1}{1+\frac{4}{\pi} \frac{1+\gamma}{1-\gamma}(1+\beta) k^{2} Q_{M}}
\end{array}
$$

Table 2. SSD technique attenuations at the resonance frequency 

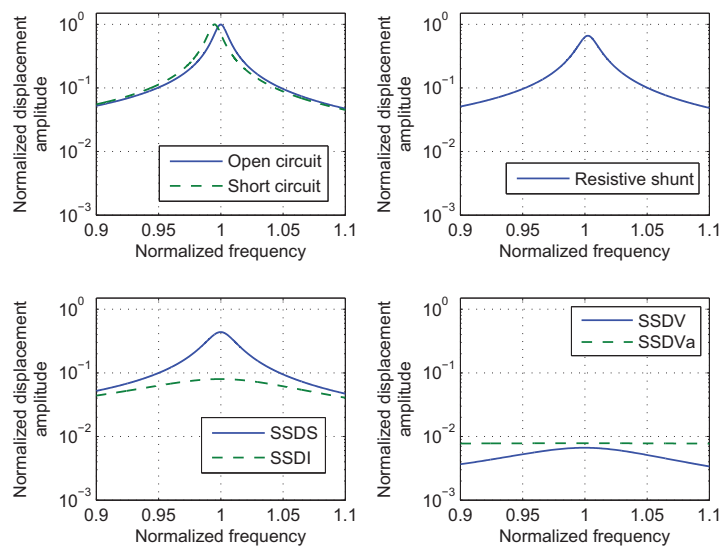

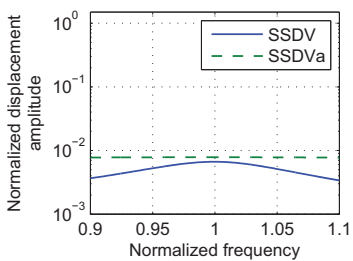

(a)

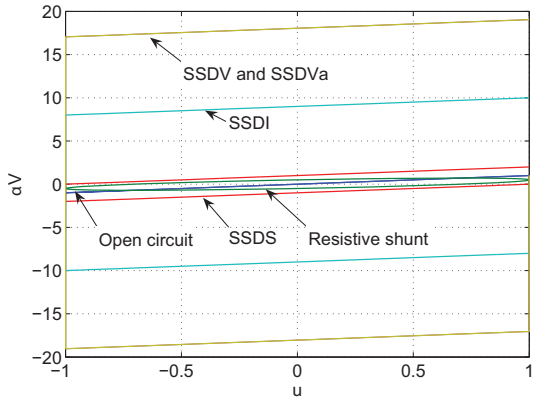

(b)

Fig. 8. (a) Frequency response functions and (b) energy cycles of SSD techniques and comparison with resistive shunt (at optimal load) $\left(M=1 \mathrm{~g}, K_{D}=1000 \mathrm{~N} . \mathrm{m}^{-1}, C=10^{-2} \mathrm{~N} . \mathrm{m}^{-2}\right.$ $\left.\left(Q_{M}=100\right), \alpha=10^{-3} \mathrm{~N} . \mathrm{V}^{-1}, C_{0}=10^{-7} \mathrm{~F}\left(k^{2}=10^{-2}\right), \gamma=0.8, V_{S}=80 \mathrm{~V}, \beta=10\right)$

the semi-active approaches (SSDV and SSDVa). The performance increase from the resistive shunting to the SSDVa can also be demonstrated by the energy cycle in the coordinate $(u$, $\alpha V$ ), derived from the converted energy (Eq. (20)), where the increase in terms of area denotes the increase in terms of converted energy, and thus performance improvement. However, it should be noted that in the case of the SSDV, the attenuation expression and transfer function are negative when:

$$
\alpha \frac{4}{\pi} \frac{1+\gamma}{1-\gamma} \frac{V_{S}}{F_{M}}>1
$$

denoting the emergence of instabilities in the system (Lallart, Badel \& Guyomar, 2008d), while the SSDVa, matching the value of the voltage source to the displacement magnitude, is unconditionally stable.

\subsection{Broadband excitation}

The previous section focused on the vibration control when only one particular mode is concerned. Thanks to the nature of the maximum and minimum detection, the adaptivity of the SSD control is ensured in the case of frequency drifts (due for example to temperature or pressure variation or ageing).

However, realistic excitation forces of structures are more likely to be random. When the structure is submitted to such forces, the nonlinear nature of the approach prevents the estimation of the system response. However, it has been shown that using a time-domain approach may help in predicting the response, by modeling the switching process as a series of step responses (Guyomar \& Lallart, 2007b; Lallart et al., 2007)5.

\footnotetext{
5 This approach also premits a more accurate prediction of the response of systems featuring low mechanical quality factor (Lallart et al., 2007).
} 


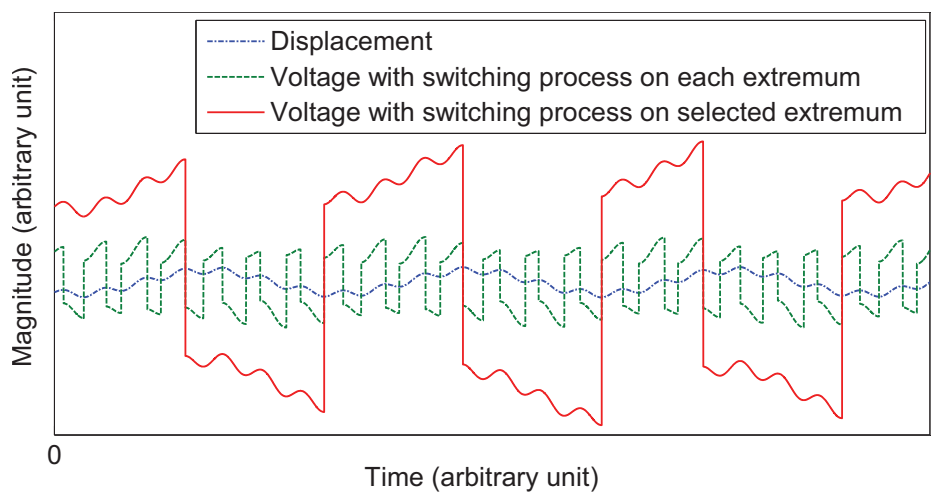

Fig. 9. SSD technique waveforms under brodband or multimodal excitation

Moreover, in the case of complex excitation, it can be shown that a trade-off exists between the voltage increase caused by the cumulative effect of the switching process itself and the number of switching events (Figure 9), as the converted energy is given by:

$$
E_{\text {conv }}=\frac{1}{2} C_{0} V^{2}+\frac{1}{2} C_{0} \sum_{k=1}^{n}\left[\left(1-\gamma^{2}\right) V_{k}^{2}-(1+\gamma)\left(2 \gamma V_{k}+(1+\gamma) V_{S}\right)\right]
$$

with $V_{k}$ referring to the voltage at the $k^{\text {th }}$ switching time instant.

Hence, if the switching process occurs on each maximum and minimum voltage values, the voltage increase would be limited due to the losses in the inversion circuit. But if the number of switching events is limited, the voltage is no longer proportional to the sign of the velocity, limiting the conversion enhancement.

In addition, Figure 9 shows that the switching process focuses on the highest mode when no particular extremum selection is performed. As higher mode are generally less coupled than first modes, this leads to moderate performance of the control.

In order to ensure the conversion gain allowed by the nonlinear process, it is therefore mandatory to properly select the voltage extrema. Several approaches can be used:

- Probabilistic approach (Guyomar \& Badel, 2006b): This method consists in computing the cumulative distribution function of the squared displacement or voltage over a given time period. Then the switching process is enabled (i.e., the inversion of the voltage will occur on the next extremum value) if the value of the squared displacement or voltage is greater than a reference value corresponding to a user-defined probability threshold.

- Statistical approach (Guyomar, Richard \& Mohammadi, 2007c): In this approach, the switching process is enabled if the signal (squared displacement or voltage) is greater than the sum of the mean value and standard deviation weighted by a user-defined coefficient. An alternative method consists in using the RMS value rather than the mean value and standard deviation.

- Analog approach (Lallart et al., 2008e): This method consists in setting the threshold using analog components (typically a lossy peak detector). Although this approach is not as precise as the previously exposed techniques, the computation of the threshold is very simple, allowing the self-powered implementation of the concept. 
- Modal approach (Harari, Richard \& Gaudiller, 2009): The principles of this control consists in filtering the response of a piezoelectric element used as sensor in order to select only one mode to control. The number of required piezoelectric actuators is equal to the number of controlled mode in this case. The technique can besides be enhanced by ensuring that the switching process occurs on actual extrema.

Hence, the principles of disabling the switching process when the control signal is less than a threshold allows controlling the trade-off between voltage increase and number of switching events (Ji et al., 2009). The performance of the vibration control system are therefore increased by a typical factor of 2 compared to the simple SSDI that consists of switching on all extrema (Guyomar \& Badel, 2006b; Guyomar, Richard \& Mohammadi, 2007c). Such an approach also permits a simultaneous control of several modes of the structure, and automatically adapts the control to the mode featuring the highest energy.

\subsection{Implementation considerations \& discussion}

When dealing with embeddable systems for vibration control, it is mandatory to take into account the integration potential of the envisaged technique. In the case of the SSD control, it has been shown that the required energy for the actuation is limited, and even null for the semi-passive approaches (SSDS and SSDI).

In particular, the control principles that consists in detecting minimum and maximum voltage values can be done in a simple way by comparing the voltage itself with its delayed version as depicted in Figure 10, giving the position of maximum values (the minimum positions detection is done using an additional detector but with inversed polarities).

The implementation of such a self-powered switching device is shown in Figure 11 (Richard, Guyomar \& Lefeuvre, 2007). It consists in comparing the voltage (using transistor $T_{2}$ ) with its delayed version obtained using a diode, a resistor and a capacitor. Once the delayed version greater than the voltage, the transistor $T_{2}$ is $\mathrm{ON}$, turning $T_{1} \mathrm{ON}$ as well. Hence the inversion process is initiated. In order to ensure that the switching process stops half an oscillation period latter, a diode $\left(D_{1}\right)$ is placed in the switching branch which stops the process when the current cancels (corresponding to the end of the inversion). Finally, it has to be noted that the capacitor $C$ used both for the delay and energy storage in order to drive the transistors should be large enough to ensure a full voltage inversion but also small enough to ensure that it is discharged at the beginning of the next positive voltage value (Figure 10).

It is also important to note that such an implementation also works using the same patch both for actuation and sensing; hence no additional piezoelectric insert is required.

Such a simple implementation, that requires widely available components, can therefore be easily embedded while having a very low cost. Such a device also have a wide frequency range of operation, covering more than a decade ${ }^{6}$.

As stated in paragraph 3.2, the SSD technique can be improved in broadband excitation conditions by using a proper extremum selection. In particular, it is possible to slightly modify the proposed implementation in order to implement a threshold that enables the maximum detection. It consists of using an envelope detector that is compared to the piezovoltage. The detection through the peak detector is then enabled only if the piezovoltage is greater than the threshold (Lallart et al., 2008e).

\footnotetext{
${ }^{6}$ It can be demonstrated that a small delay between the actual occurrence of a maximum value and the switching process does not have a significant impact on the performance of the system.
} 


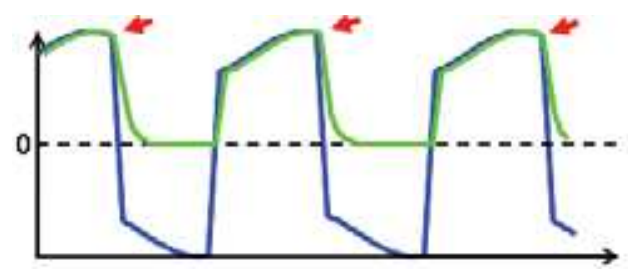

Fig. 10. Maximum detection using delayed signal

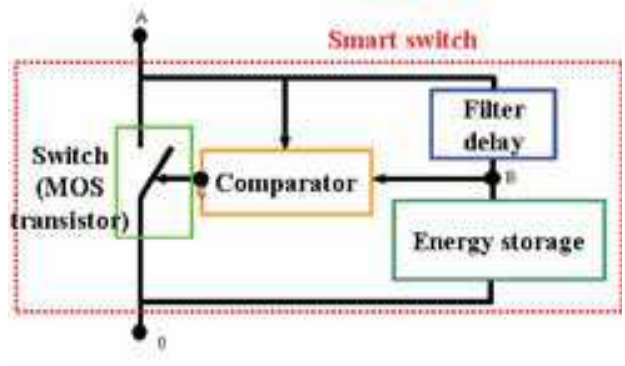

(a)

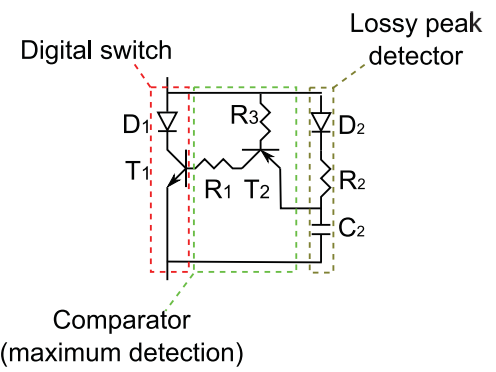

(b)

Fig. 11. Principles of the self-powered maximum detection and unipolar circuit schematic (Richard, Guyomar \& Lefeuvre, 2007)
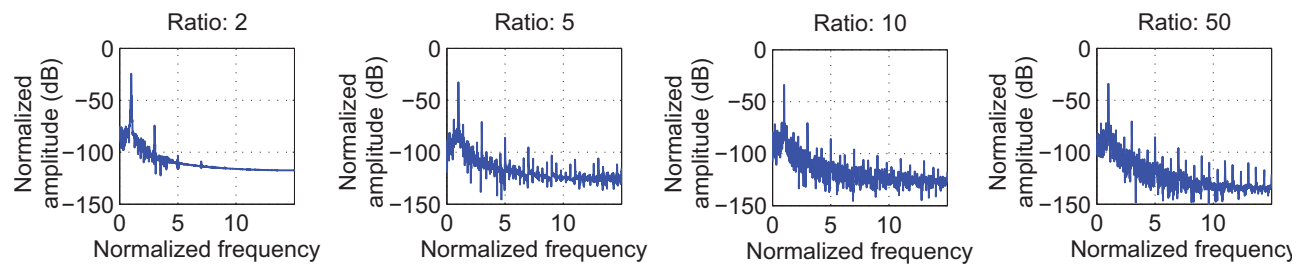

Fig. 12. Frequency response functions (normalized w.r.t. maximal magnitude of the uncontrolled system) for different switching frequencies (normalized w.r.t. vibration frequency)

An additional concern about the implementation of the SSD technique lies in the fact that the additional piecewise voltage (which is a crenel function under monochromatic excitation) leads to the appearance of odd harmonics, which may be harmful if a higher mode matches one of these harmonics, or when performing a sound wave control for comfort (as the human hearing system is more sensitive to higher frequencies). In this case, it is possible to increase the switching time period (for example by using an inductance with a higher value). This strategy hence allows a reduction of the harmonics (Petit et al., 2004) without decreasing significantly the performance of the damping system (Figure 12).

Finally, it can be noted that the switch principles are independent from the physical quantity. Hence, it is possible to extend the control to magnetic actuators (Lallart et al., 2008b) by controlling the current rather than the voltage and by operating dual transformations of the control circuit and law (i.e., normally closed switch in parallel with the actuator). 


\section{Blind Switch Damping (BSD) approaches}

The basic concept of the SSD techniques lies in switching synchronously with the structure motion. Therefore, it is mandatory to monitor the voltage or displacement (although the detection may be simply realized as shown in the previous section). The purpose of this section is to expose another nonlinear approach that allows limiting the vibrations by artificially increasing the mechanical losses without any needs of synchronization (Lallart et al., 2009). Hence no voltage or displacement sensing is required. Because of this structureindependent and non-synchronous nature, this concept is called Blind Switch Damping on Short-circuit (BSDS).

\subsection{Principles}

The principles of the BSDS technique consists in switching the piezoelectric element on short circuit (Figure 13) with a frequency $f_{s} n$ times higher than the vibration frequency $f_{0}$ :

$$
f_{s}=n f_{0}, n \in \mathfrak{R}^{+} \text {and } n \gg 1 .
$$

Hence, this intermittent short-circuit switching approach allows shaping the voltage so that this latter is in phase with the speed thanks to a low-cost differentiation process, hence leading to viscous losses and therefore reducing the vibrations.

\subsection{Performance under monochromatic excitation}

The principles of the BSDS technique consist in dissipating the electrostatic energy available on the piezoelectric element ${ }^{7}$. The expression of the piezovoltage when using the BSDS method, given as:

$$
V=\frac{\alpha}{C_{0}}\left[u(t)-u\left(k \frac{T_{0}}{n}\right)\right] \text { for } k \frac{T_{0}}{n} \leq t<(k+1) \frac{T_{0}}{n} \text { with } k \in \mathbb{N},
$$

with $T_{0}$ the vibration period $\left(T_{0}=1 / f_{0}\right)$, shows the differentiation performed by this approach. Considering that the switching coefficient $n$ is far greater than 1 and that the electromechanical structure filters frequencies higher than its resonance frequency, the piezoelectric voltage may be approximated by its first harmonic such as:

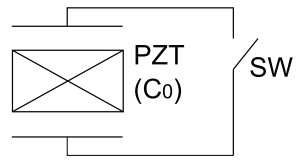

(a)

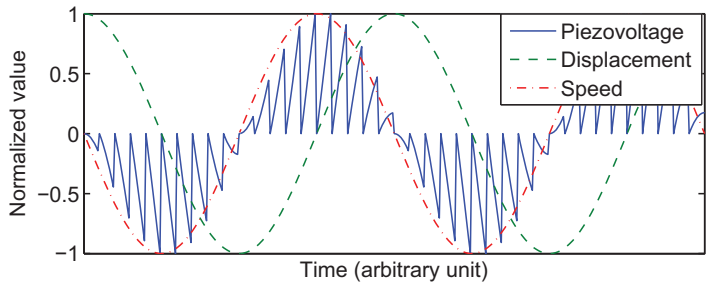

(b)

Fig. 13. (a) BSDS implementation and (b) typical waveforms

\footnotetext{
7 The cancellation of the piezovoltage may also be seen as a change in the initial conditions.
} 


$$
V \approx \frac{\alpha}{C_{0}} \frac{T_{0}}{\pi n} \dot{u}(t)
$$

Hence, inserting this equation in the spring-mass-damper model obtained in Section 2.1 (Eq. (14)) leads to the motion equation when using the BSDS:

$$
M \ddot{u}+\left(C+\frac{\alpha^{2}}{C_{0}} \frac{T_{0}}{\pi n}\right) \dot{u}+K_{E} u=F,
$$

which demonstrates the viscous damping effect performed by the method (while the SSD techniques are equivalent, in a mechanical point of view, to a dry friction). Hence, the attenuation at the resonance frequency permitted by the BSDS is given by:

$$
A_{B S D S}=\frac{1}{1+\frac{1}{C} \frac{\alpha^{2}}{C_{0}} \frac{T_{0}}{\pi n}}=\frac{1}{1+\frac{2}{n} k^{2} Q_{M}} .
$$

From this expression it can be shown that the BSDS technique efficiency is inversely proportional to the switching coefficient $n$ (Figure 14). Hence a trade-off appears between a correct reconstruction of the speed (high values of $n$ ) and the performance (moderate values of $n$ ).

\subsection{Performance enhancement using voltage sources}

As shown in the previous subsection, the performance of the BSDS technique are tightly related to the inverse switching coefficient $1 / n$. As this coefficient should be large enough to ensure a proper speed reconstruction, this would lead to a moderate attenuation effect.

As the voltage is already in phase with the speed, the conversion enhancement allowing an artificial increase of the squared coupling coefficient $k^{2}$ (leading to higher damping abilities as shown in Figure 14) has to be performed by increasing the piezovoltage. Such operation may be obtained by adding voltage sources in the switching device (making the technique no longer semi-passive however) as depicted in Figure 15, leading to the concept of Blind Switch Damping on Voltage sources (BSDV).

The differentiation operation is still performed using short-circuit switching (through branch 1 in Figure 15(a)), but the next switching process in this case aims at magnifying the piezovoltage in order to have a higher damping ability (through branch 2 in Figure 15(a)). Hence, while the switching frequency is still given by $n f_{0}\left(S_{1}\right)$, the system intermittently switches on short-circuit and voltage source. The addition of the inductance $L$ allows a better energy transfer from the voltage source to the piezoelectric element. In addition, the voltage source $V_{S}$ is matched to the sign of the piezovoltage:

$$
V_{S}=\left|V_{S}\right| \operatorname{sign}(V) \text {. }
$$

\footnotetext{
8 In this section it will be considered that the uncontrolled case correspond to a short-circuited piezoelectric element. Such an assumption allows much simpler expressions while having a negligible impact on the performance evaluation.
} 


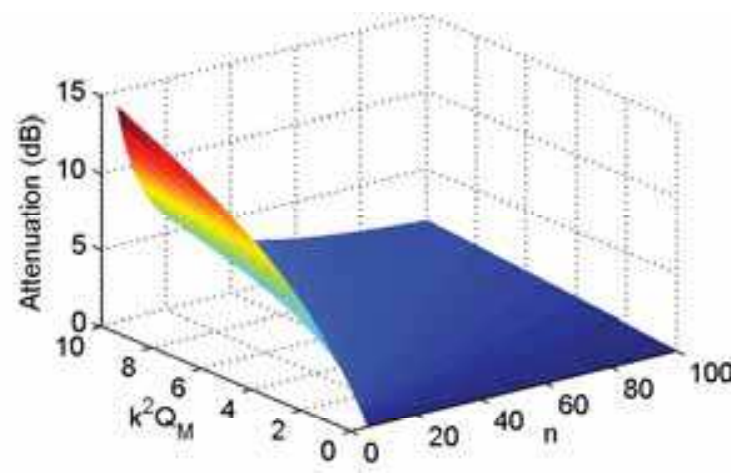

Fig. 14. Performance of the BSDS technique as a function of the product of the squared coupling coefficient by the mechanical quality factor $k^{2} Q_{M}$ and switching coefficient $n$

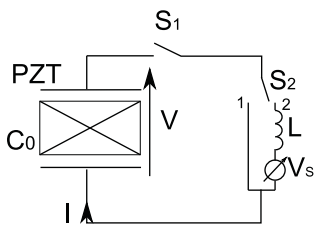

(a)

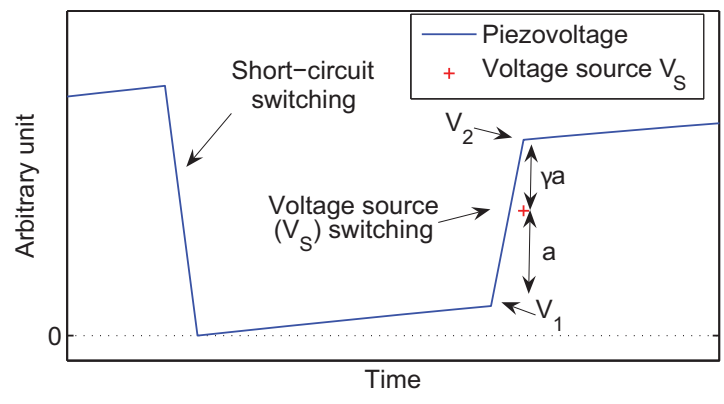

(b)

Fig. 15. (a) BSDV implementation and (b) typical waveforms

The value of the voltage $V_{1}$ before the voltage increase process is given by the differentiation performed by the short-circuit switching:

$$
V_{1}=\frac{\alpha}{C_{0}}\left[u\left((2 k+1) \frac{T_{0}}{n}\right)-u\left(2 k \frac{T_{0}}{n}\right)\right] \text { with } k \in \mathbb{N},
$$

while the voltage after the voltage switching yields:

$$
V_{2}=\gamma\left(V_{S}-V_{1}\right)+V_{S}
$$

Hence, assuming that the structure filters high harmonics, the piezoelectric voltage may be approximated by:

$$
\begin{array}{ll}
V(t) \approx \frac{\alpha}{C_{0}} \frac{T_{0}}{n} \dot{u}(t) & \text { for } 2 k \frac{T_{0}}{n} \leq t<(2 k+1) \frac{T_{0}}{n} \quad(k \in \mathbb{N}) \\
& \text { (short-circuit switching) } \\
V(t) \approx \gamma\left(V_{S}-\frac{\alpha}{C_{0}} \frac{T_{0}}{n} \dot{u}(t)\right)+V_{S} & \text { for }(2 k+1) \frac{T_{0}}{n} \leq t<2(k+1) \frac{T_{0}}{n} \quad(k \in \mathbb{N}) \\
& \text { (voltage source switching) }
\end{array}
$$




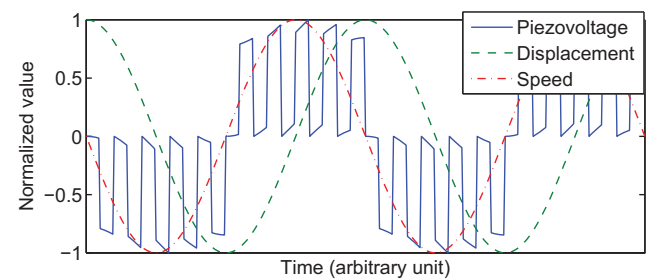

(a) BSDVp

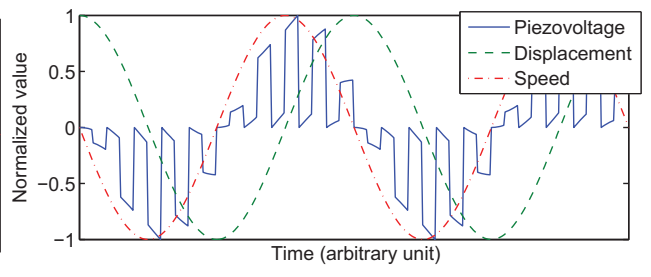

(b) BSDVa

Fig. 16. BSDV typical waveforms

The value of the voltage source $V_{S}$ can then be defined in several ways. In the followings, two cases will be considered:

- The voltage source $V_{S}$ is proportional to the displacement magnitude $u_{M}$, leading to the technique called Blind Switch Damping on piecewise constant Voltage source (BSDVp), as the voltage source value is changed on maximum displacement only.

- $\quad$ The voltage source $V_{S}$ is proportional to the voltage value $V_{1}$ just before the voltage source switching process $V_{1}$, leading to the technique called Blind Switch Damping on adaptive Voltage source (BSDVa), as the voltage source continuously matches the piezovoltage (except during the inversion).

The associated waveforms to each of these approaches are depicted in Figure 16. It can be observed that the piezovoltage in the BSDVp case has an envelope that is proportional to the sign of the speed, while the envelope of the BSDVa technique is proportional to the speed, hence featuring less harmonics.

\subsubsection{Blind Switch Damping on piecewise constant Voltage source (BSDVp)}

In the case of the BSDVp control, the voltage source value is given as:

$$
V_{S}=\beta \frac{\alpha}{C_{0}} \operatorname{sign}(V(t)) \approx \beta \frac{\alpha}{C_{0}} \operatorname{sign}(\dot{u}(t)),
$$

leading to the expression of the voltage, assuming that $n \gg 1$ and $\beta \gg 1$ :

$$
\begin{cases}V(t) \approx 0 & \text { for } 2 k \frac{T_{0}}{n} \leq t<(2 k+1) \frac{T_{0}}{n} \quad(k \in \mathbb{N}) \\ & \text { (short-circuit switching) } \\ V(t) \approx(1+\gamma) V_{S}=(1+\gamma) \beta \frac{\alpha}{C_{0}} \operatorname{sign}(\dot{u}(t)) & \text { for }(2 k+1) \frac{T_{0}}{n} \leq t<2(k+1) \frac{T_{0}}{n} \quad(k \in \mathbb{N}) \\ & \text { (voltage sources witching) }\end{cases}
$$

Hence, if the structure filters higher harmonics, the motion equation turns to:

$$
M \ddot{u}+\left(C+\frac{2}{\pi} \frac{\alpha^{2}}{C_{0}} \frac{1}{\omega}(1+\gamma) \beta\right) \dot{u}+K_{E} u=F,
$$

with $\omega$ the angular frequency. The associated attenuation at the resonance frequency is therefore given by: 


$$
A_{B S D V p}=\frac{1}{1+\frac{2}{\pi} \frac{1}{C \omega_{0}} \frac{\alpha^{2}}{C_{0}}(1+\gamma) \beta}=\frac{1}{1+\frac{2}{\pi}(1+\gamma) \beta k^{2} Q_{M}} .
$$

This expression shows that indexing the value of the voltage on the displacement magnitude allows having an attenuation that is independent from the switching coefficient $n$. In addition, the BSDVp technique is close to the SSD technique, except that the crenel function is modulated by another crenel function of frequency $n f_{0}$ (Figure 16(a)). Because of this modulation (which cancels the voltage half the time), the magnitude of the first harmonic of the piezovoltage is divided by two compared to a SSD technique featuring the same voltage magnitude.

\subsubsection{Blind Switch Damping on piecewise adaptive Voltage source (BSDVa)}

When indexing the voltage source value $V_{S}$ to the voltage $V_{1}$ just before the switching process such as:

$$
V_{S}=\beta V_{1}=\beta V\left((2 k+1) \frac{T_{0}}{n}\right), k \in \mathbb{N},
$$

the expression of the piezovoltage may be approximated by (assuming $n \gg 1$ and $\beta \gg 1$ ):

$$
\left\{\begin{array}{ll}
V(t) \approx 0 & \text { for } 2 k \frac{T_{0}}{n} \leq t<(2 k+1) \frac{T_{0}}{n} \quad(k \in \mathbb{N}) \\
& \text { (short-circuit switching) } \\
V(t) \approx(1+\gamma) V_{S}=(1+\gamma) \beta \frac{\alpha}{C_{0}} \frac{T_{0}}{n} \dot{u}(t) & \text { for }(2 k+1) \frac{T_{0}}{n} \leq t<2(k+1) \frac{T_{0}}{n} \quad(k \in \mathbb{N}) \\
& \text { (voltage source switching) }
\end{array} \quad(k\right.
$$

The first harmonic approximation of this voltage is then given by:

$$
V \approx(1+\gamma) \beta \frac{\alpha}{C_{0}} \frac{T_{0}}{\pi n} \dot{u}(t),
$$

yielding the transfer function of the displacement in the frequency domain and expression of the attenuation at the resonance frequency:

$$
\begin{aligned}
\frac{U(\omega)}{F(\omega)} & =\frac{1}{-M \omega^{2}+j\left(C+(1+\gamma) \beta \frac{\alpha^{2}}{C_{0}} \frac{T_{0}}{\pi n}\right) \omega+K_{E}}, \\
A_{B S D V a} & =\frac{1}{1+(1+\gamma) \beta \frac{1}{C} \frac{\alpha^{2}}{C_{0}}}=\frac{1}{1+\frac{2}{n}(1+\gamma) \beta k^{2} Q_{M}} .
\end{aligned}
$$

This expression shows that the attenuation in this case is similar to the one obtained using the BSDS technique (Eq. 37), but with a value of the squared coupling coefficient artificially increase by a factor $(1+\gamma) \beta$, leading to higher damping abilities as shown in Figure 14. In addition, the voltage waveforms of the BSDVa may be seen as a modulation of a voltage 
proportional to the speed (as for a simple derivative control) by a crenel function at a frequency $n f_{0}$.

\subsection{Discussion}

One of the main advantage of the BSD techniques over the SSD method is not only the nonsynchronized approach (which allows a wider practical frequency range of operation), but also the possibility of controlling the reinjected harmonics through the switching coefficient $n$. In particular, as the switching frequency is far greater than the vibration frequency, the reinjected harmonics of the BSD techniques are far away from the first mode frequencies (contrary to the SSD case where odd harmonics appear). This is however not the case for the BSDVp as it features an envelope that is a piecewise constant voltage in phase with the speed as in the SSD case.

However, it should be noted that BSD techniques have to be semi-active in order to dispose of significant damping abilities. Nevertheless, the BSDV techniques can be made extremely low-power, by using the energy harvesting scheme described by Lefeuvre et al. (2005) during the short-circuit switching, hence allowing the recovery of most of the supplied energy during the voltage source switching process.

Among the exposed BSD methods, the BSDVa technique seems to be the most interesting one, allowing a significant damping behavior (contrary to the BSDS method that is limited), while preserving a good trade-off between performance and required energy. It can also be noted that the BSDVp technique may present instabilities for high values of $n$ (Lallart et al., 2009).

Finally, the concept of the BSD techniques based on a low-cost differentiation of the displacement by intermittently switching the piezoelement is also well adapted to broadband $\operatorname{control}^{9}$, as the switching process allows the low-cost reconstruction of the speed as long as the switching frequency is much higher than the highest vibration frequency. The BSD principles may also be seen as a spreading of the converted mechanical energy on a wide frequency range (spillover) because of the switching process. Hence, as the structure filters this frequency contents, a damping effect is achieved.

\section{Synchronized Switch Stiffness Control (SSSC)}

The principles of the previously exposed techniques aimed at reducing the vibrations by increasing, in a mechanical point of view, the losses in the mechanical structure. However, another strategy for reducing the vibration magnitude consists in ensuring that the structure is excited out of its resonance, hence preventing the energy from entering into the structure.

\subsection{Principles}

The purpose of the concept exposed here is to modify the stiffness through a simple and low-cost nonlinear treatment similar to the SSD techniques. The main difference is that the switching process occurs on zero displacement (Guyomar, Lallart \& Monnier, 2008), as shown in Figure 1710.

\footnotetext{
${ }^{9}$ In the case of the BSDVp technique, the definition of displacement amplitude is however delicate in the case of random vibrations.

10 As the voltage is switched on zero displacement values, the use of an external voltage source is mandatory to perform a voltage inversion; hence, the SSSC technique is semi-active.
} 


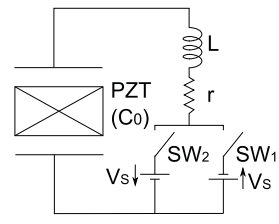

(a)

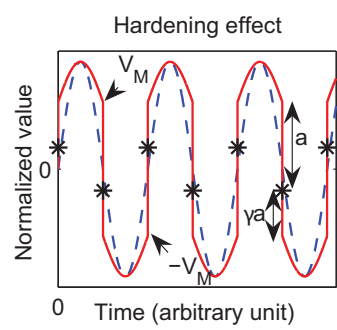

Fig. 17. (a) SSSC schematics and (b) typical waveforms

Due to the voltage switch on zero displacements, the voltage may be decomposed into one voltage proportional to the displacement, and a piecewise constant voltage in phase with the displacement which modifies the stiffness of the system. As this latter may be tuned through the voltage source $V_{S}$, its effect (and thus the equivalent stiffness of the system) may be controlled.

\subsection{Performance under monochromatic excitation}

In the case of a monochromatic excitation, the piezoelectric voltage in the case of the SSSC technique may be decomposed as:

$$
V=\frac{\alpha}{C_{0}} u+V_{M} \operatorname{sign}(u),
$$

where $V_{M}$ refers to the amplitude of the crenel function (Figure 17(b)), given by:

$$
V_{M}-V_{S}=\gamma\left(V_{M}+V_{S}\right) \Rightarrow V_{M}=\frac{1+\gamma}{1-\gamma} V_{S}
$$

The absolute value of $V_{S}$ being matched on the displacement magnitude such as:

$$
\left|V_{S}\right|=|\beta| \frac{\alpha}{C_{0}} u_{M}
$$

The user-defined coefficient $\beta$ may be positive or negative, according to the type of desired effect (hardening or softening effect - Figure 17(b)). Hence, using the first harmonic approximation (i.e., the structure filters the higher harmonics), the piezovoltage equals to:

$$
V \approx \frac{\alpha}{C_{0}}\left(1+\beta \frac{4}{\pi} \frac{1+\gamma}{1-\gamma}\right) u .
$$

Merging this expression in the motion equation yields the equivalent stiffness of the system:

$$
K_{\mathrm{SSSC}}=\left(K_{D}+\frac{4}{\pi} \frac{\alpha^{2}}{C_{0}} \frac{1+\gamma}{1-\gamma} \beta\right)=K_{D}\left(1+\frac{4}{\pi} \frac{1+\gamma}{1-\gamma} \beta k^{2}\right)
$$




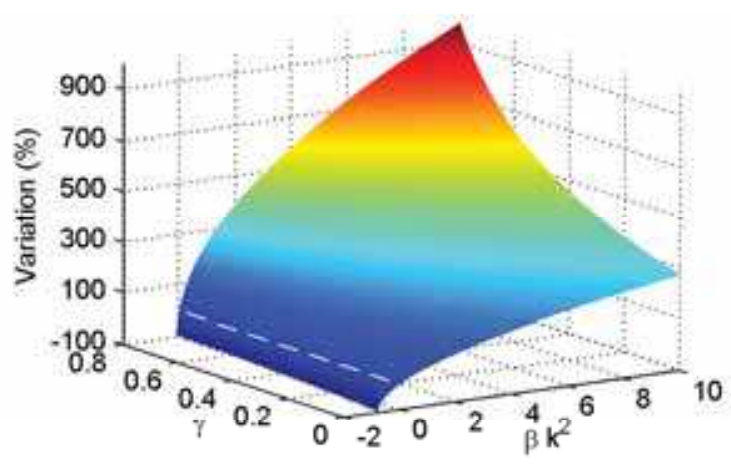

Fig. 18. Resonance frequency shift using the SSSC approach (dashed line represents null stiffness change)

It can be seen that, contrary to the previous techniques, the mechanical quality factor $Q_{M}$ does not intervene in the expression of the equivalent stiffness. It is also possible to express the change operated by the SSSC technique in terms of resonance frequency and mechanical quality factor:

$$
\left\{\begin{array}{l}
\left(\omega_{r e s}\right)_{S S S C}=\omega_{r e s} \sqrt{1+\frac{4}{\pi} \frac{1+\gamma}{1-\gamma} \beta k^{2}} \\
\left(Q_{M}\right)_{S S S C}=Q_{M} \sqrt{1+\frac{4}{\pi} \frac{1+\gamma}{1-\gamma} \beta k^{2}}
\end{array} .\right.
$$

The performance of the system in terms of resonance frequency change is depicted in Figure 18, showing that the SSSC method allows both decreasing or increasing the resonance frequency, with a pseudo-quadratic behavior as a function of $\gamma$, while the change becomes less significant as $\beta k^{2}$ increases.

\subsection{Energy requirements}

Several techniques can be used for tuning the stiffness of a system (Wickramasinghe et al., 2004; Nitzsche et al., 2005), the most common being a proportional control. However, in the framework of embedded devices, such a control should consume a very few amount of energy. Hence, the purpose of this section is to compare the energy requirements of the SSSC method with respect to a classical proportional control.

In the latter case, the control voltage is set to be proportional to the displacement such as:

$$
\mathrm{V}_{\text {proportional }}=K_{C} u \text {, }
$$

with $K_{C}$ the tuning coefficient. Hence, the equivalent stiffness yields:

$$
K_{\text {proportional }}=K+\alpha K_{C} .
$$

When using proportional control, the main source of losses is the heat dissipated in the parallel parasitic resistance $r_{P}$ of the piezoelectric element. Therefore, the dissipated power yields: 


$$
P_{\text {proportional }}=f_{0} \int_{0}^{f_{0}} \frac{V_{\text {proportional }}^{2}}{r_{P}} d t=\frac{K_{C}}{2 r_{P}} u_{M}{ }^{2} .
$$

In the case of the SSSC control, the losses in the parasitic resistance may be neglected facing the losses in the switching circuit (the piezoelectric element is in open-circuit most of the time). Hence, the required power for the SSSC control is given by:

$$
P_{\mathrm{SSSC}}=2 f_{0} \int_{-V_{M}}^{V_{M}} C_{0} V_{S} d V=4 f_{0} \frac{1+\gamma}{1-\gamma} \frac{\alpha^{2}}{C_{0}} \beta^{2} u_{M}^{2} .
$$

Figure 19 depicts the power required for both techniques as a function of the stiffness variation. The power has been normalized with respect to the power required to have a $100 \%$ stiffness variation using the proportional control. This figure clearly demonstrates the ability of the SSSC approach for efficiently controlling the stiffness of the system without requiring high power; compared to the proportional control, the power requirement is typically 10 times less.

\subsection{Application to vibration damping}

The purpose of this section is the application of the SSSC technique for limiting the vibrations of the structure. Unlike the previously exposed methods whose principles are to artificially increase the mechanical losses, the concept proposed here consists in shifting the resonance frequency far away from the frequency spectrum of the input force.

The principles of such a control rely on frequency sensing (e.g., based on zero crossing detection - Figure 20). Then, from the frequency information, the value of the tuning coefficient $\beta$ is chosen such that the resonance frequency of the structure is out of resonance (for example, the absolute value of $\beta$ may be fixed and its sign obtained from the frequency, as depicted in Figure 20(b)).

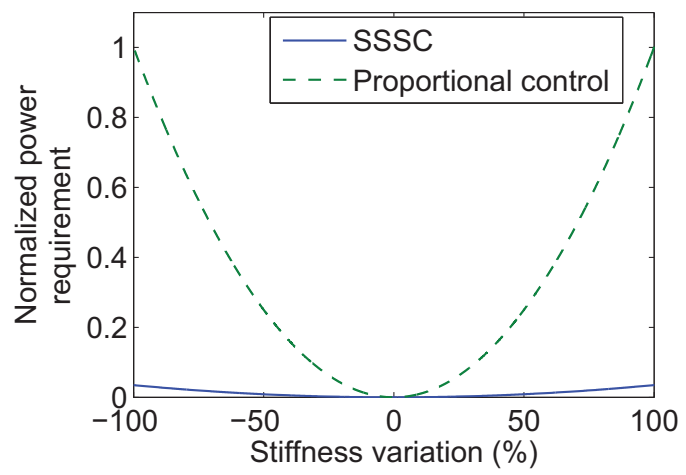

Fig. 19. Normalized power requirements for the SSSC technique $(\gamma=0.8)$ and comparison with proportional control $\left(1 /\left(2 \pi r_{P} C_{0} f_{0}=0.05\right)\right.$ at $\left.1 \mathrm{kHz}\right)$ for a vibration frequency of $f_{0}=20$ $\mathrm{Hz}$ 


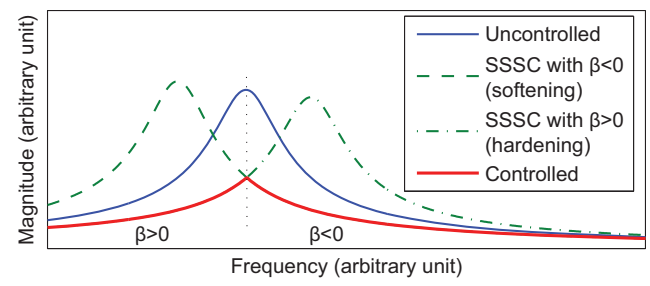

(a)

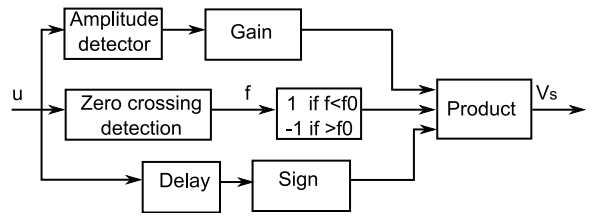

(b)

Fig. 20. SSSC technique applied to vibration damping: (a) principles; (b) implementation example

\section{Conclusion}

This chapter exposed energy-efficient methods for vibration control using piezoelectric elements. Based on a nonlinear approach that consists in quickly changing the voltage of piezoelectric elements, it has been shown that such a concept allows an efficient control of the vibrations while requiring a very few amount of power, therefore making the method suitable for embedded systems.

In a first step, the principles of the switching technique have been exposed, showing the conversion increase, thanks to a voltage magnification by a cumulative process as well as a reduction of the phase between the voltage and velocity.

Then this concept has been directly applied to the reduction of the vibrations, leading to the so-called Synchronized Switch Damping (SSD) methods. Four techniques have been exposed, whether the voltage switching is done on a short-circuit (SSDS), an inductor (SSDI), an inductor in series with constant voltage sources (SSDV), or an inductor in series with adaptive voltage sources (SSDVa); the two formers being possibly self-powered as no external energy is required for the control. The analysis of the performance under a monochromatic excitation showed that the attenuation at the resonance frequency obtained using such method is tightly related to the mechanical quality factor and squared coupling coefficient, with a typical reduction of the vibrations around $20 \mathrm{~dB}$ for the SSDI and $40 \mathrm{~dB}$ for the SSDV and SSDVa. The application of the SSD method to broadband excitations has also been discussed, showing the trade-off between voltage increase and number of switching events, that can be controlled by selecting proper switching time instants (extremum selection). Finally, the realistic implementation of the technique to integrated systems has been demonstrated, as well as the application of the concept to electromagnetic actuators.

The second part of this chapter consisted in exposing another technique for vibration damping by a non-synchronized approach called Blind Switch Damping (BSD). Based on a low-cost differentiation obtained by switching the piezoelectric element on a short-circuit at a frequency far greater than the vibration frequency, the technique allows reconstructing the speed with the voltage, leading to viscous damping effect. However, the performance of such a method remains modest ( $\mathrm{a}$ few $\mathrm{dB}$ ), and increasing the voltage is mandatory to get significant damping abilities. This voltage magnification can be obtained using voltage sources that can either be proportional to the displacement magnitude or to the value of the voltage just before the switching process. The low-cost differentiation is still obtained by connecting the piezoelectric insert on a short-circuit, but the next switching process consists in increasing the voltage, allowing damping abilities close to the SSD approach. The two 
main advantages of the BSD methods are the possibility of controlling the reinjected harmonics as well as the independency from the host structure. Another interesting aspects of the BSDV methods is the possibility of working in a energy injection/energy recovery basis that dramatically limits the required power.

Finally, a third technique has been introduced. Contrary to the SSD and BSD approaches that consist in artificially increasing the mechanical losses in the structure, the proposed concept, named Synchronized Switch Stiffness Control (SSSC), allows tuning the stiffness in a low-cost way (typically 10 times less energy is required for the same effect compared to a proportional control). The principle of this method is to connect the piezoelectric element on a resonant electrical network featuring a voltage source each time the displacement is null. Hence, such an approach shapes a piecewise constant voltage in phase with the displacement, hence modifying the stiffness. An application to vibration reduction by ensuring that the structure is excited out of its resonance through stiffness tuning has also be exposed.

The performance comparison of all the exposed techniques is presented in Table 3. From this table, it can be seen that the SSDI featuring threshold detection is particularly interesting when no external energy is supplied to the system as it allows a self-powered broadband control with significant damping abilities, while the BSDVa approach, thanks to its harmonic reinjection control, broadband approach, effectiveness and low-power consumption, is a good option when a few amount of energy can be provided to the system. It can however be noted that the combination of several methods is possible, for example using the SSD control together with a stiffness tuning, hence allowing an increase of the global performance. Finally, the combination of active control methods with the exposed approaches for limiting the power requirements while achieving important damping if necessary (Harari, Richard \& Gaudiller, 2009) may also be envisaged.

\begin{tabular}{|c|c|c|c|c|c|}
\hline $\begin{array}{l}\text { Technique } \\
\text { (Kind) }\end{array}$ & Efficiency & Adaptiveness & Robustness & $\begin{array}{c}\text { Power } \\
\text { requirements }\end{array}$ & $\begin{array}{l}\text { Implementation } \\
\text { easiness }\end{array}$ \\
\hline SSDS (semi-passive) & & 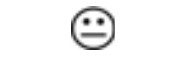 & $(:)$ & $(-)$ & $(-)$ \\
\hline SSDI (semi-passive) & $\ddot{\theta}$ & $\ddot{\theta}$ & & 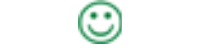 & \\
\hline $\begin{array}{l}\text { SSDI with threshold } \\
\text { detection (semi-passive) }\end{array}$ & 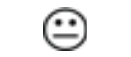 & $(\ddot{)}$ & 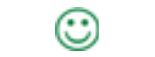 & $(-)$ & $(-)$ \\
\hline SSDV (semi-active) & () & $\ominus$ & & 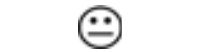 & (ت) \\
\hline SSDVa (semi-active) & & 8 & & $\because \cdots$ & \\
\hline BSDS (semi-passive) & & (2) & & $\because$ & 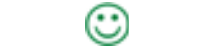 \\
\hline BSDVp (semi-active) & & $\ddot{\theta}$ & $(\ddot{-})$ & $\ddot{\theta}$ & $\ddot{-}$ \\
\hline BSDVa (semi-active) & & & & & \\
\hline SSSC (semi-active) & (9) & $\Theta$ & $(-)$ & $\ddot{\theta}$ & $\ddot{\theta}$ \\
\hline
\end{tabular}

Table 3. Comparison of the exposed techniques

11 ;) means that the method can be self-powered. 


\section{References}

Badel, A.; Sebald, G.; Guyomar, D.; Lallart, M.; Lefeuvre, E.; Richard, C. \& Qiu, J. (2006). Piezoelectric vibration control by synchronized switching on adaptive voltage sources: Towards wideband semi-active damping. el Acoust. Soc. Am., Vol. 119, No. 5, 2815-2825.

Badel, A.; Lagache, M.; Guyomar, D.; Lefeuvre, E. \& Richard, C. (2007). Finite Element and Simple Lumped Modeling for Flexural Nonlinear Semi-passive Damping. \& Intell. Mater. Syst. Struct., Vol. 18, 727-742.

Clark, W. W. (2000). Vibration control with state-switching piezoelectric material, eI Intell. Mater. Syst. Struct., Vol. 11, 263-271.

Cunefare, K. A. (2002). State-switched absorber for vibration control of point-excited beams, II Intell. Mater. Syst. Struct., Vol. 13, 97-105.

Davis, C. L. \& Lesieutre, G. A. (2000). An actively Tuned Solid-State Vibration Absorber Using Capacitive Shunting of Piezoelectric Stiffness, I of Sound and Vibration, Vol. 232, No. 3, 601-617.

Fleming, A. J. \& Moheimani, S. O. R. (2003). Adaptive Piezoelectric Shunt Damping, Smart Mater. Struct., Vol. 12, 36-48.

Gerhold, C.H. (1989). Active Control of Flexural Vibrations in Beams, cburnal of Aerospace Engineering, Vol. 2, No. 3, 141-154.

Guyomar, D.; Badel, A.; Lefeuvre, E. \& Richard, C. (2005). Towards energy harvesting using active materials and conversion improvement by nonlinear processing, IEEE Trans. Ultrason., Ferroelect., Freq. Contr., Vol. 52, 584-595.

Guyomar, D.; Faiz, A.; Petit, L. \& Richard, C. (2006a). Wave reflection and transmission reduction using a piezoelectric semipassive nonlinear technique. I Acoust. Soc. Am., Vol. 119, No. 1,285-298.

Guyomar, D. \& Badel, A. (2006b). Nonlinear semi-passive multimodal vibration damping: An efficient probabilistic approach. I Sound Vib., Vol. 294, 249-268.

Guyomar, D.; Jayet, Y.; Petit, L.; Lefeuvre, E.; Monnier, T.; Richard, C. \& Lallart, M. (2007a) Synchronized Switch Harvesting applied to Self-Powered Smart Systems : Piezoactive Microgenerators for Autonomous Wireless Transmitters, Sens. Act. A: Phys., Vol. 138, No. 1, 151-160. doi : 10.1016/j.sna.2007.04.009

Guyomar, D. \& Lallart, M. (2007b). Piezo-transducer Modelling with a Switched Output Voltage: Application to Energy Harvesting and Self-Powered Vibration Control. Proceedings of the 19th International Congress on Acoustics (ICA), 2-7 September 2007, Madrid, Spain.

Guyomar, D.; Richard, C. \& Mohammadi, S. (2007c). Semi-passive random vibration control based on statistics. I Sound Vib., Vol. 307, 818-833.

Guyomar, D.; Lallart, M. \& Monnier, T. (2008). Stiffness Tuning Using a Low-Cost SemiActive NonLinear Technique, IEEE/ASME Transactions on Mechatronics, Vol. 13, No. 5, 604- 607.

Harari, S.; Richard, C. \& Gaudiller, L. (2009). New Semi-active Multi-modal Vibration Control Using Piezoceramic Components. el Intell. Mater. Syst. Struct., Vol. 20, No. 13, 1603- 1613.

Holdhusen, M. H. \& Cunefare, K. A. (2003). Damping Effects on the State-Switched Absorber Used for Vibration Suppression, II Intell. Mater. Syst. Struct., Vol. 14, No. 9, 551-561. doi:10.1177/104538903036919 
Inman, D. J.; Ahmadian, M. \& Claus, R. O. (2001). Simultaneous Active Damping and Health Monitoring of Aircraft Panels, dI Intell. Mater. Syst. Struct., Vol. 12, No. 11, 775-783. doi: $10.1177 / 104538901400438064$

Ji, H.; Qiu, J.; Zhu, K.; Y. Chen \& Badel, A. (2009). Multi-modal vibration control using a synchronized switch based on a displacement switching threshold, Smart Mater. Struct., Vol. 18, 035016.

Johnson, C. D. (1995). Design of Passive Damping Systems, el of Mechanical Design, Vol. 117, No. B, 171-176.

Lallart, M.; Guyomar, D.; Petit, L.; Richard, C.; Lefeuvre, E. \& Guiffard, B. (2007). Effect Of Low Mechanical Quality Factor On Non-Linear Damping And Energy Harvesting Techniques, Proceedings of the 18th International Conference on Adaptive Structures and Technologies (ICAST), 3-5 October 2007, Ottawa, Ontario, Canada.

Lallart, M.; Guyomar, D.; Jayet, Y.; Petit, L.; Lefeuvre, E.; Monnier, T.; Guy, P. \& Richard, C. (2008a). Synchronized Switch Harvesting applied to Selfpowered Smart Systems: Piezoactive Microgenerators for Autonomous Wireless Receiver, Sens. Act. A: Phys., Vol. 147, No. 1, 263-272. doi: 10.1016/j.sna.2008. 04.006.

Lallart, M. \& Guyomar, D. (2008b). Optimized Self-Powered Switching Circuit for NonLinear Energy Harvesting with Low Voltage Output, Smart Mater. Struct., Vol. 17, 035030. doi: 10.1088/0964-1726/17/3/035030

Lallart, M.; Garbuio, L.; Petit, L.; Richard, C. \& Guyomar, D. (2008c) Double Synchronized Switch Harvesting (DSSH) : A New Energy Harvesting Scheme for Efficient Energy Extraction, IEEE Trans. Ultrason., Ferroelect., Freq. Contr., Vol. 55, No. 10, 2119-2130.

Lallart, M.; Badel, A. \& Guyomar, D. (2008d). Non-Linear Semi-Active Damping Using Constant Or Adaptive Voltage Sources: A Stability Analysis. I Intell. Mat. Syst. Struct., Vol. 19, No. 10, 1131-1142. doi : 10.1177/1045389X07083612

Lallart, M.; Lefeuvre, E.; Richard, C. \& Guyomar, D. (2008e). Self-Powered Circuit for Broadband, Multimodal Piezoelectric Vibration Control. Sens. Act. A: Phys., Vol. 143, No. 2, 277-382, 2008. doi : 10.1016/j.sna.2007.11.017

Lallart, M.; Magnet, C.; Richard, C.; Lefeuvre, E.; Petit, L.; Guyomar, D. \& Bouillault, F. (2008f). New Synchronized Switch Damping Methods Using Dual Transformations. Sens. Act. A: Phys., Vol. 143, No. 2, 302-314. doi : 10.1016/j.sna.2007.12.001

Lallart, M.; Harari, S.; Petit, L.; Guyomar, D; Richard, T.; Richard, C. \& Gaudiller, L. (2009). Blind Switch Damping (BSD): A Self-Adaptive Semi-Active Damping Technique. $J$ Sound Vib., Vol. 329, Nos. 1-2, 29-41.

Lefeuvre, E.; Badel, A.; Richard, C. \& Guyomar, D. (2005). Piezoelectric energy harvesting device optimization by synchronous electric charge extraction. I Intell. Mat. Syst. Struct., Vol. 16, No. 10, 865-876.

Lefeuvre, E.; Badel, A.; Richard, C.; Petit, L. \& Guyomar, D. (2006a). A comparison between several vibration-powered piezoelectric generators for standalone systems, Sens. Act. A: Phys, Vol. 126, 405-416.

Lefeuvre, E.; Badel, A.; Petit, L.; Richard, C. \& Guyomar D. (2006b). Semi-passive Piezoelectric Structural Damping by Synchronized Switching on Voltage Sources. I Intell. Mater. Syst. Struct., Vol. 17, Nos. 8-9, 653-660.

Lesieutre, G. A. (1998). Vibration Damping and Control using Shunted Piezoelectric Materials, Shock and Vib. Digest, Vol. 30, pp. 187-195. 
Nitzsche, F.; Harold, T.; Wickramasinghe, V. K.; Young, C. \& Zimcik, D. G. (2005). Development of a Maximum Energy Extraction Control for the Smart Spring, $J$ Intell. Mater. Syst. Struct., Vol. 16, Nos. 11-12, 1057-1066.

Makihara, K.; Onoda, J. \& Minesugi, K. (2007). A self-sensing method for switching vibration suppression with a piezoelectric actuator, Smart Mater. Struct., Vol. 16, 455-461.

Petit, L.; Lefeuvre, E.; Richard, C. \& Guyomar, D. (2004). A broadband semi passive piezoelectric technique for structural damping, Proceedings of SPIE conference on Smart Struct. Mater. 1999: Passive Damping and Isolation, San Diego, CA, USA, March 2004, Vol. 5386, 414-425. ISBN : 0-8194-5303-X

Qiu, J. H. \& Haraguchi M. (2006). Vibration control of a plate using a self-sensing actuator and an adaptive control approach, el Intell. Mater. Syst. Struct., Vol. 17, 661-669.

Richard, C.; Guyomar, D.; Audigier, D. \& Ching, G. (1999). Semi passive damping using continuous switching of a piezoelectric device, Proceedings of SPIE conference on Smart Struct. Mater. 1999: Passive Damping and Isolation, Newport Beach, CA, USA, March 1999, Vol. 3672, 104-111. ISBN : 0-8194-3146-X

Richard, C.; Guyomar, D.; Audigier, D. \& Bassaler, H. (2000). Enhanced semi passive damping using continuous switching of a piezoelectric device on an inductor, Proceedings of SPIE conference on Smart Struct. Mater. 2000: Passive Damping and Isolation, Newport Beach, CA, USA, March 2000, Vol. 3989, 288-299. ISBN : 0-81943607-0

Richard C.; Guyomar, D. \& Lefeuvre, E. (2007). Self-Powered Electronic Breaker With Automatic Switching By Detecting Maxima Or Minima Of Potential Difference Between Its Power Electrodes, patent \# PCT/FR2005/003000, publication number: WO/2007/063194, 2007.

Veley, D. E. \& Rao, S. S. (1996). A Comparison of Active, Passive and Hybrid Damping in Structural Design, Smart Mater. Struct., Vol. 5, 660 - 671.

Wickramasinghe, V. K.; Young, C.; Zimcik, D. G.; Harold, T. \& Nitzsche, F. (2004). Smart Spring, a Novel Adaptive Impendance Control Approach for Active Vibration Suppression Applications, Proceedings of SPIE Smart Materials and Structures: Smart Structures and Integrated Systems, San Diego, CA, USA, March 2004, Vol. 5390.

Wu, S. Y. (1998). Method for multiple shunt damping of structural vibration using a single PZT transducer, Proceedings of SPIE conference on Smart Struct. Mater. 1998: Passive Damping and Isolation, San Diego, CA, USA, March 1998, Vol. 3327, 159-168. 


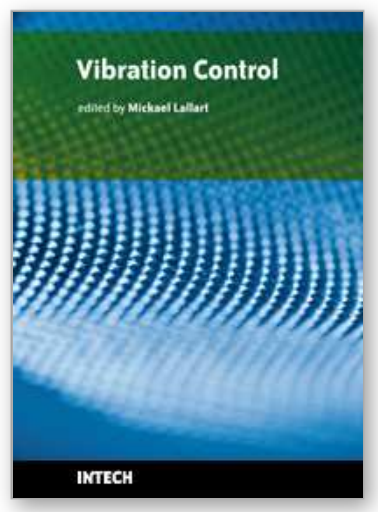

\author{
Vibration Control \\ Edited by MickaÃfÂथl Lallart
}

ISBN 978-953-307-117-6

Hard cover, 380 pages

Publisher Sciyo

Published online 18, August, 2010

Published in print edition August, 2010

Vibrations are a part of our environment and daily life. Many of them are useful and are needed for many purposes, one of the best example being the hearing system. Nevertheless, vibrations are often undesirable and have to be suppressed or reduced, as they may be harmful to structures by generating damages or compromise the comfort of users through noise generation of mechanical wave transmission to the body. the purpose of this book is to present basic and advanced methods for efficiently controlling the vibrations and limiting their effects. Open-access publishing is an extraordinary opportunity for a wide dissemination of high quality research. This book is not an exception to this, and I am proud to introduce the works performed by experts from all over the world.

\title{
How to reference
}

In order to correctly reference this scholarly work, feel free to copy and paste the following:

Mickael Lallart and Daniel Guyomar (2010). Self-Powered and Low-Power Piezoelectric Vibration Control Using Nonlinear Approaches, Vibration Control, MickaÃfÂAl Lallart (Ed.), ISBN: 978-953-307-117-6, InTech, Available from: http://www.intechopen.com/books/vibration-control/self-powered-and-low-power-piezoelectricvibration-control-using-nonlinear-approaches

\section{INTECH}

open science | open minds

\section{InTech Europe}

University Campus STeP Ri

Slavka Krautzeka 83/A

51000 Rijeka, Croatia

Phone: +385 (51) 770447

Fax: +385 (51) 686166

www.intechopen.com

\section{InTech China}

Unit 405, Office Block, Hotel Equatorial Shanghai

No.65, Yan An Road (West), Shanghai, 200040, China

中国上海市延安西路65号上海国际贵都大饭店办公楼405单元

Phone: +86-21-62489820

Fax: +86-21-62489821 
(C) 2010 The Author(s). Licensee IntechOpen. This chapter is distributed under the terms of the Creative Commons Attribution-NonCommercialShareAlike-3.0 License, which permits use, distribution and reproduction for non-commercial purposes, provided the original is properly cited and derivative works building on this content are distributed under the same license. 